\title{
Quadratic LYM-type inequalities for intersecting Sperner families
}

\author{
Christian Bey
}

Fakultät für Mathematik, Otto-von-Guericke-Universität, Universitätsplatz, 2, 39106 Magdeburg, Germany email: christian.bey@mathematik.uni-magdeburg.de

Let $\mathcal{F} \subseteq 2^{[n]}$ be a intersecting Sperner family (i.e. $A \not \subset B, A \cap B \neq \emptyset$ for all $A, B \in \mathcal{F}$ ) with profile vector $\left(f_{i}\right)_{i=0 \ldots n}$ (i.e. $f_{i}=\left|\mathcal{F} \cap\left(\begin{array}{c}{[n]} \\ i\end{array}\right)\right|$ ). We present quadratic inequalities in the $f_{i}$ 's which sharpen the previously known linear LYM-type inequalities.

Keywords: Sperner family, antichain, LYM inequality

Let $[n]$ denote the set $\{1,2, \ldots, n\}, 2^{[n]}$ the powerset of $[n]$, and $\left(\begin{array}{c}{[n]} \\ i\end{array}\right)$ the set of all $i$-element subsets of $[n]$. Given a set system $\mathcal{F} \subseteq 2^{[n]}$, put $f_{i}=\left|\mathcal{F} \cap\left(\begin{array}{c}{[n]} \\ i\end{array}\right)\right|$ and call $\left(f_{i}\right)_{i=0 \ldots n}$ the profile vector of $\mathcal{F}$.

Recall that a set $\mathcal{F} \subseteq 2^{[n]}$ is called a Sperner family (or antichain) if there are no inclusions among the members of $\mathcal{F}: A \not \subset B$ for all $A, B \in \mathcal{F}$. The well-known theorem by Sperner (cf. [2]) says that the maximum size of an antichain in $2^{[n]}$ is $\left(\begin{array}{c}n \\ \lfloor n / 2\rfloor\end{array}\right)$, the only families achieving this cardinality being $\left(\begin{array}{c}{[n]} \\ \lfloor n / 2\rfloor\end{array}\right)$ and $\left(\begin{array}{c}{[n]} \\ \lceil n / 2\rceil\end{array}\right)$.

A generalization of Sperner's Theorem is the well-known LYM inequality (cf. [2]): If $\mathcal{F} \subseteq 2^{[n]}$ is a Sperner family with profile vector $\left(f_{i}\right)_{i=0 \ldots n}$ then

$$
\sum_{i=0}^{n} \frac{f_{i}}{\left(\begin{array}{c}
n \\
i
\end{array}\right)} \leq 1
$$

In [1] we have sharpened the LYM inequality by adding to its LHS all possible products of fractions $f_{i} /\left(\begin{array}{c}n \\ i\end{array}\right)$ with suitable coefficients. A special case of this sharpened version is the following quadratic LYM inequality $(Q L Y M)$ :

Theorem 1 ([1]) Let $\mathcal{F} \subseteq 2^{[n]}$ be a Sperner family with profile vector $\left(f_{i}\right)_{i=0 \ldots n}$. Then

$$
\sum_{0 \leq i \leq n} \frac{f_{i}}{\left(\begin{array}{c}
n \\
i
\end{array}\right)}+\sum_{0<i<j<n} \frac{n(j-i)}{i(n-j)} \frac{f_{i}}{\left(\begin{array}{c}
n \\
i
\end{array}\right)} \frac{f_{j}}{\left(\begin{array}{c}
n \\
j
\end{array}\right)} \leq 1 .
$$

The proof of Theorem 1 was based on the well-known Kruskal-Katona Theorem (cf. [2]) and induction on $n$.

1365-8050 @ 2005 Discrete Mathematics and Theoretical Computer Science (DMTCS), Nancy, France 
Here we continue these investigations by presenting such sharpened LYM-type inequalities for the class of intersecting Sperner families. Recall that a set system $\mathcal{F} \subseteq 2^{[n]}$ is called intersecting if $A \cap B \neq \emptyset$ for all $A, B \in \mathcal{F}$.

The following LYM-type inequality for intersecting Sperner families $\mathcal{F} \subseteq 2^{[n]}$ was found in [3]:

$$
\sum_{i \leq n / 2} \frac{f_{i}}{\left(\begin{array}{c}
n \\
i-1
\end{array}\right)}+\sum_{j>n / 2} \frac{f_{j}}{\left(\begin{array}{c}
n \\
j
\end{array}\right)} \leq 1
$$

We have the following sharpening:

Theorem 2 Let $\mathcal{F} \subseteq 2^{[n]}$ be a intersecting Sperner family with profile vector $\left(f_{i}\right)_{i=0 \ldots n}$. Put

$$
c_{i, j}= \begin{cases}\frac{n(j-i)}{(i-1) j} & \text { if } i<j \leq n / 2 \\ \frac{n(j-i)}{(n-i+1)(n-j)} & \text { if } n / 2<i<j \\ \frac{n(n+1-i-j)}{(i-1) j} & \text { if } i \leq n / 2<j, i+j \leq n+1 \\ \frac{n(i+j-n-1)}{(n-i+1)(n-j)} & \text { if } i \leq n / 2<j, i+j \geq n+1 .\end{cases}
$$

Then

$$
\sum_{i \leq n / 2} \frac{f_{i}}{\left(\begin{array}{c}
n \\
i-1
\end{array}\right)}+\sum_{j>n / 2} \frac{f_{j}}{\left(\begin{array}{c}
n \\
j
\end{array}\right)}+\sum_{i<j} c_{i, j} \frac{f_{i}}{\left(\begin{array}{c}
n \\
i-1
\end{array}\right)} \frac{f_{j}}{\left(\begin{array}{c}
n \\
j
\end{array}\right)} \leq 1 .
$$

The proof of Theorem 2 is based on the previous one and the following connection between profile vectors of Sperner - and intersecting Sperner families.

Lemma 1 Let $\left(f_{i}\right)_{i=0 \ldots n}$ be the profile vector of an intersecting Sperner family in $2^{[n]}$. Then $\left(g_{i}\right)_{i=0 . . n}$ defined by

$$
g_{i}= \begin{cases}f_{i+1}+f_{n-i} & \text { if } i<\frac{n-1}{2} \\ f_{i+1} & \text { if } i=\frac{n-1}{2} \\ 0 & \text { if } i>\frac{n-1}{2}\end{cases}
$$

is profile vector of a Sperner family in $2^{[n]}$.

Besides (1), other LYM-type inequalities for intersecting Sperner families are known. In fact, in $[4,5]$ the convex hull of all profile vectors of such set systems was determined. In our talk we will also address the question on sharpening all facet inequalities of this convex hull.

\section{References}

[1] C. Bey, Polynomial LYM inequalities. Combinatorica 25 (2005), 19-38.

[2] B. Bollobás, Combinatorics. Cambridge Univ. Press, Cambridge 1986.

[3] C. Greene, Gy.O.H. Katona and D.J. Kleitman, Extensions of the Erdős-Ko-Rado theorem. SIAM Stud. Appl. Math 55 (1976), 1-8.

[4] P.L. Erdős, P. Frankl and Gy.O.H. Katona, Intersecting Sperner families and their convex hulls. Combinatorica 4 (1984), 21-34. 
[5] Gy.O.H. Katona and G. Schild, Linear inequalities describing the class of intersecting Sperner families of subsets, in: R. Bodendiek and R. Henn, eds, Topics in Combinatorics and Graph Theory (Physica-Verlag, Heidelberg, 1990), 413-420. 
